\title{
Vegetarianism and Health Status among the Senior Citizens of Iligan City
}

\author{
Cherlita C. Cudal
}

Corazon T. Biong

\author{
College of Education \\ MSU-lligan Institute of Technology \\ Iligan City, Philippines
}

\section{Doi:10.5901/mjss.2013.v4n9p586}

\begin{abstract}
This study aimed to find out the vegetarianism and health status among selected senior citizens of Iligan City using a survey research design that makes use of the descriptive-comparative method of research. The results of the study are used as a basis for creating a health intervention program that will provide the respondents an option of a better and healthy life style. Out of the 100 elderly respondents, 70 are non-vegetarians and 30 are vegetarians. The results showed that vegetarian diet has a positive effect on the respondents' muscular endurance and muscular strength. The result also showed that vegetarian diet which is less in saturated fats and high in fiber increases the cardiovascular endurance of the respondents, thus, improving their personality type, stress resiliency level, and physical activity participation. Furthermore, the vegetarian diet has shown to improve the senior citizens' medical condition, but the effects of aging in inevitable. Lifestyle diseases have been observed with the vegetarian respondents because of aging and it is also observed the same with the non-vegetarian respondents. In conclusion, vegetarian diet is good since it promotes a healthy life especially among the elderly.
\end{abstract}

Keywords: vegetarianism, diet, health status, lifestyle

\section{Introduction}

Today's world of industrialization and automation resulted to a fast-paced society which adversely affected the health and life longevity of mankind. The unhealthy lifestyle of the century as evidenced by many people's wrong choice of food consumption and inactivity is considered to be the culprit of man's shorter life expectancy at present. The lives of man are becoming more and more comfortable with what technology has offered them. They prefer to use the electronic driven machines rather than use the muscles to accomplish daily tasks. Thus, they become less and less active. Most interestingly, they prefer to eat processed foods loaded with cholesterol and sugar which are conveniently available in grocery stores and in fast-food chains rather than prepare a fresh, simple, yet delicious and nutritious plant-based meal packed with phytochemicals, vitamins and minerals. Obviously, people are now eating less healthy kinds of food. Unhealthy lifestyle may lead to having certain diseases. Lifestyle diseases like obesity, diabetes mellitus, hyperlipidemia, hypertension, coronary artery disease and cancer are becoming common in all walks of life nowadays. Ornish (1993), stated that "our lifestyle choices: what we eat, how we respond to stress, lack of exercise, smoking and so on are the major causes of heart diseases and other debilitating illnesses." To have more productive years in life, one must have a healthy body. A healthy body however, does not mean the absence of sickness, but it is the development of a man's physical, mental, social and spiritual dimensions, which are integral part of life. There are many important factors to have a healthy body. Specifically, in this study, vegetarianism is believed to affect the health status among the senior citizens of lligan City. It is likewise believed that a healthy diet with fruits and vegetables enables the elderly to have a healthy status that is free from diseases and having very good cardiovascular endurance, flexibility, muscular endurance, and muscular strength which are the health-related physical fitness components of this study.

\section{Literature Review}

Vegetarianism is a practice of following a diet based on plant-based foods including fruits, vegetables, cereal grains, nuts, and seeds, with or without dairy products and eggs. Vegetarians do not eat meat, game, poultry, fish, crustacea, shellfish, or products of animal slaughter such as animal-derived gelatin and rennet. Vegetarianism is a way of achieving a better health. It is a kind of diet consisting of plant-based foods including fruits, vegetables, cereals, grains, nuts and 
seeds with or without dairy products and eggs. It has five categories which are fruitarian, macrobiotic, vegan, lactovegetarian and lacto-ovo vegetarian (Cox, 2002). Fruitarian is a diet consisting of raw or dried fruits, nuts, seeds, honey and vegetable oil. The Macrobiotic excludes all animal foods, dairy products and eggs. It uses only unprocessed, unrefined, 'natural' and 'organic' cereals, grains and condiments such as miso and seaweed. Vegan excludes all animal food, dairy products and eggs. In the purest sense, excludes all animal products including honey, gelatin, silk, wool, leather and animal-derived food additives. The Lacto-vegetarian excludes all animal foods and eggs but include milk and milk products. Lastly, Lacto-ovo-vegetarian which excludes all animal foods, however, it includes milk, milk products and eggs. A vegetarian diet is known to confer a wide range of health benefits. Research has shown vegetarians to suffer less heart disease, hypertension, obesity, diabetes, various cancers, diverticular disease, bowel disorders, gall stones, kidney stones,andosteoporosis. The American Dietetic Association and Dietitians of Canada have stated: "Vegetarian diets offer a number of nutritional benefits, including lower levels of saturated fat, cholesterol, and animal protein as well as higher levels of carbohydrates, fiber, magnesium, potassium, folate, and antioxidants such as vitaminsC and $\mathrm{E}$ and phytochemicals.

According to Melina, Davis and Stanton (2005), health is the main reason why people chose to be vegans. While a vegetarian diet reduces one's risk of chronic, degenerative diseases such as coronary artery disease, cancer, diabetes, obesity, osteoporosis, gall bladder disease and hypertension, the plant-based diet minimizes diseases and meets the current recommendations percentage of fats, carbohydrate and protein than an omnivore diet. Rakesh (2000), stated that it was common belief that non-vegetarian diet is superior to vegetarian diet. People used to think that non-vegetarian diet provides more power to the body and it is full of energetic ingredients. This theory attracted more and more people and ultimately a large number of people of the world adopted non-vegetarian diet. According to the new developments in medical sciences, while vegetarian diet is closer to and is more useful for human nature; and is more scientific for the human body, the non-vegetarian diet contains cholesterol and saturated fatty acid which are the root causes of problems like coronary heart disease, cerebro-vascular accidents (strokes), eye diseases and high blood pressure. In a nonvegetarian diet, only $60 \%$ of its content is useful for human body, the remaining $40 \%$ contains harmful and toxic products. In addition, the non-vegetarian diet is generally heavy for stomach and produces acidity, which in turn can cause many diseases of gastrointestinal system. However, the concept of vegetarianism is changing. While the vegetarian diet is the panacea for modern lifestyle, Segasothy and Phillips (1999), stated that the intake of vitamin B12 is lower in vegetarian diets and deficiencies in this vitamin have been reported to cause irreversible nerve deterioration. In a similar light, Phillips (2005) added that calcium absorption is reported to decline with age among elderly vegetarians and there is reduced availability of minerals such as iron, copper and zinc in vegan diets which are important in immunity. Dworkin (1999), stated that the disproportionate consumption of foods high in fats, often at the expense of foods high in complex carbohydrates and fiber- such as vegetables, fruits, and whole-grain products- that may be more conducive to health. He said that the more an individual restricts his or her diet, the more difficult it is to get all the nutrients that are needed. He added that the inadequate supply of vitamin $D$ and calcium cause rickets, iron deficiency causes anemia and the lack of protein can brought hair loss. Ewan (2002), also added that vegetarian respondents were of equal fitness, but the vegetarians scored lower on standing long jump and 30-s sit ups than the non-vegetarians. Moreover, the heart rate of the vegetarians young adults recovered substantially faster following the step test. As a dependent variable of the study, many believe that vegetarians have negative effects on physical fitness health -related component. However, literature suggests that the heart rate of a vegetarian adult recovered substantially faster following the Step test in the cardiovascular endurance test.

These pieces of literature had prompted the researcher to find out the relationship between vegetarianism and health status among the elderly who are officially registered at the Office of the Senior Citizens Affairs (OSCA) lligan City. It is hoped that the results would determine whether those vegetarians are more physically fit than the non-vegetarians in terms of the health-related components such as cardio-vascular endurance, flexibility, muscular endurance, and muscular strength. The results of the study moreover, would reveal the medical conditions of the senior citizens in terms of the presence and absence of the lifestyle diseases they may be suffering. Furthermore, this study looks into the effects of the moderating variables and to help explain the findings of the study, considering the factors such as age, gender, socio-economic status, educational attainment, personality type, stress resiliency level, physical activity participation, and vices that the elderly are involved with. Lastly, this study is thought to be very significant because this aims to provide the elderly insights on the positive and negative effects of vegetarianisms on life longevity.

Aging is a gradual process that reflects the influence of genetics, lifestyles, and environment over the course of the life span. Beginning the age of 30 , the purpose of cell creation changes, no longer supplying new cells to replace old cells. At older ages, this process of cell replication slows ever more, and the effects of aging on body organs begin to 
appear. The effect of nutrient intake, is mediated by lifestyle behaviors including physical activity, stress, smoking, alcohol consumption, and exposure to environmental factors.

Emotional health may be affected by poor eating habits, resulting in hypoglycemia or low blood glucose levels. Low blood glucose occurs normally in anyone who is physically hungry. When the body's need for food is ignored (e.g. missing meals because of poor planning or are too busy to eat), feeling of anxiety and confusion and trembling may occur. Emotions may be harder to control when one feels this way (DeYoung et al., 2005). They added that elderly may struggle with the deaths of family members and friends and adjustment to retirement. Although some delight in retirement, others view retirement as a loss of social status. This combination of death and loss of status may lead to isolation and depression.

One of the early studies on the relationship of exercise to aging was done by Dr. Herbert de Vries. In one study of his more than 200 men and women, ages 56 California retirement communities, participated in a fitness program that included walking, a walk-jog routine, calisthenics, and stretching. After just six weeks, their blood pressure dropped, body fat decreased, maximum oxygen transport increased, and neuromuscular signs of nervous tension diminished. Other studies have shown that moderate or vigorous exercise can reduce blood cholesterol levels. And this is an important factor, for in atherosclerosis patients this level is often higher than it should be (Vance et al., 2004).

In the study conducted by Hutton (1994), it showed that smokers look older than non-smokers. After 25 years, women who smoke appears to decrease blood supply to the skin, cutting off its normal supply of oxygen. In an experiment carried out in California, it was revealed that one study of nearly 600 smokers at the National Heart, Lung and Blood Institute found more damage in the lungs of women aged 35 to 44 than in the lungs of men of the same age. With an already shortened life expectancy, these relatively young women had only the prospect of steadily worsening health ahead if they persisted with their habit (Hutton, 1994).

Alejandro (2006) stated that the consumption of meat and other animal products has been conclusively linked to heart attacks, strokes, diabetes, obesity, and cancer in the Philippines today. According to the Department of Health, this causes "chronic uncontrolled states and progressive organ damage leading to death." The Philippine Cancer Society urges Filipinos to increase their intake of these wholesome foods, and the World Health Organization has declared that inadequate consumption of fruits and vegetables is among the top 10 selected risk factors for global mortality. Furthermore, Dugang (2001) observed that Filipinos are not really vegetable eaters. "Only about 10 percent of the average Filipino diet is composed of vegetables. She cautioned that vegetarian diet should start from the parents in order to teach and inform their children about the benefits of vegetarian diets.

\section{Methodology}

This section deals with the methods that the researcher used in the study. These include the research design, respondents of the study, sampling techniques, research instruments, data gathering procedures, and statistical treatment.

\subsection{Research Design}

This study made use of descriptive-comparative method of research to compare the health-related fitness components such as cardiovascular endurance, flexibility, muscular endurance, and muscular strength between the vegetarian and non-vegetarian elderly who were officially registered at the Office of the Senior Citizens Affairs (OSCA), lligan City in the fiscal year 2010.

\subsection{Respondents and Research Locale of the Study}

The respondents of this study were the officially registered senior citizens at the Office of the Senior Citizens Affairs (OSCA) of lligan City. Of the eighteen thousand two hundred twenty five $(18,225)$ registered elderly, only one hundred fifty-three (153) senior citizens were purposively selected. These one hundred fifty-three (153) respondents were the representatives of each senior citizen chapter from the forty four (44) barangays of Iligan City. There are fifty-one (51) chapters having three (3) representatives consisting of the president, vice-president, and secretary of the chapter. These number of respondents actually decreased because during the actual data gathering, only those elderly who were willing to be part of the study and were capable of executing the health-related fitness tests such as the cardiovascular test, flexibility test, muscular endurance and muscular strength tests were the only ones who became part of the respondents 
of the study. Moreover, since the independent variable is the type of respondents: vegetarians or non-vegetarians, the three (3) officer representatives in each chapter were replaced by a qualified respondent to ensure the adequacy of respondents in every group. Furthermore, the number of respondents still decreased due to absenteeism of the senior citizens during their monthly assembly at the Office of the Senior Citizens Affairs (OSCA) of lligan City every first Wednesday of the month.

\subsection{Instrument of the Study}

This study made use of a self-made questionnaire, interview, and standardized tests. The structured questionnaire was used to generate data on the profile of the respondents. On the other hand, an interview was conducted to gather first hand information about the health condition of the elderly. This was to ensure accurate information about the lifestyle diseases the elderly were suffering from. Moreover, to obtain the physical fitness health-related status of the elderly, the standardized tests for 2 Minute Step in Place Test, Sit and Reach Flexibility Test, Sit up Test, and Push up Test were used to measure the cardiovascular endurance, flexibility, muscular endurance, and muscular strength. The stress resiliency of the respondents was measured using the standardized resiliency test of Siebert (2006).

\subsection{Statistical Tools and Treatment of Data}

To analyze the data gathered from this study, the following statistical tools were used: (1) Frequency Count and Percentage. This was used to summarize the profile of the students; (2) Arithmetic Mean. This statistics was used to describe the respondents' profile into a single coefficient; (3) T-test. This was used to determine whether a significant difference exists between health status of respondents when grouped to vegetarian and non-vegetarian; Spearman's rho - This non-parametric test was used to measure linear relationship between two variables.

\section{Results and Discussion}

This part displays the presentation and analysis of data gathered during the conduct of the study. The results of the study are mostly expressed in percentages and means. Moreover, the relationships between the variables are analyzed using Spearman rho and the difference was analyzed using t-test. The profile of the respondents in this study consists of the following areas: Age, Gender, Socio-Economic Status, Educational Attainment, Personality Type, Stress Resiliency Level, Physical Activity Participation, and Vices.

\subsection{Age and Gender Profile}

More than half of the respondents were between $67-80$ years old of age. Their average age was 72 years old. As the number of aging person increases in our society, the government and other welfare organizations must be able to assess the health status of our Senior Citizen. Out of 100 respondents $62 \%$ were males; $38 \%$ were females. Since the respondents of the study were the president, vice-president and secretary of every Senior Citizen chapter in every community in lligan City, it was found out that majority of the Senior Citizens have masculine preference in selecting leaders among them.

\subsection{Socioeconomic Profile}

Majority of the respondents belonged to the very low income group (73\%). Sixteen $16 \%$ belonged to low income group and $11 \%$ belonged to middle income group. The average monthly income of the respondents was $P 6,300.00$ and it was skewed to the left. It is further revealed that low income groups tend to consume less fruits and vegetables than higherincome groups especially at present when prices of basic commodities are soaring so high. Out of 100 respondents, $40 \%$ of the respondents were either college level or college graduate; $21 \%$ of them were high school graduate and more than half (57\%) were elementary level/graduate to high school graduate. There is a substantial number of respondents who had reached college or even college graduates. It can be deduced that knowledge on healthy living is generally understood by them. 


\subsection{Personality Type}

Majority of the respondents were cardiac prone (65\%), 13\% were having high cardiac risk, $12 \%$ were a mixture of $A B$, $6 \%$ were having non-cardiac traits, and 3\% were less cardiac prone. Using the Siebert Personality Test (2006), it can be deduced that majority of the respondents were prone to cardiac arrest.

\subsection{Stress Resiliency Level}

As regards to the stress resiliency level of the respondents, more than half or sixty-six of the respondents were either very resilient or were better than most, $20 \%$ of them were slow, but adequate, $8 \%$ were struggling, and $6 \%$ needed help. Using the Resiliency Test of Siebert (2006), it can be deduced that majority of the respondents at this age were resilient in terms of handling problems or situations in life. This can be attributed to the number of years they have lived that as senior citizens they have already passed through difficult situations in life and they were able to overcome them. The ninety-four $94 \%$ of the respondents were engaged in regular physical activity; while $6 \%$ were not. The result suggested that majority of the respondents had active lifestyles with regards to regular physical activities. In the physical activities of the respondents walking was ranked first; second was gardening/farming; third was jogging and fourth was swimming. The ability to walk in normal condition enabled them to continue to perform walking as their top physical activity, this is mainly because it is the easiest form of exercise in stretching the body's muscles.

\subsection{Physical Activity Participation}

In terms of doing physical activities, $73 \%$ of the respondents were doing it daily, $16 \%$ were doing it every other day, and $11 \%$ were doing it $3 x$ a week. Since walking was the respondents' number one physical exercise, it can be deduced based on the result that most of them were doing this exercise daily, as this is the easiest form of exercise for the elderly. Moreover, the $50 \%$ of them were doing their exercises for 15 minutes per session; another 34\% were doing it for 1 hour per session and $16 \%$ were doing it for 30 minutes per session. Based on the result, majority of the respondents were doing the physical activity daily from 15 minutes to 30 minutes. It is a good sign and a positive indication that the respondents are healthy and that as studies show, exercise can really lead to better health and longer life.

\subsection{Vices of the Respondents}

In reference to the respondents' answer on whether they were drinking alcoholic beverages or not, $41 \%$ of the nonvegetarians were alcohol drinkers while $64 \%$ were not. For the vegetarian respondents, $80 \%$ of them were not alcohol drinkers while $20 \%$ were drinkers. The results suggest that there are more respondents who are non-alcohol drinkers than those who do. Out of 29 non-vegetarians who drank alcohol beverages, $65 \%$ of them only drank alcoholic drinks once in a while, $28 \%$ of them were moderate drinkers, and $7 \%$ were hard drinkers. Only 4 vegetarian respondents drank only once in a while. The results suggest that most of the respondents, who drink, did it only once in a while. The study of Faloon (2006), found out that Seventh-day Adventist who followed vegetarian diet that is free from tobacco and alcohol live significantly longer than those who eat meat and have vices.

There were $17 \%$ of the non-vegetarian respondents smoked cigarettes while $8 \%$ did not. For the vegetarian respondents, $80 \%$ of them did not smoke while $20 \%$ did. The results show that majority of the non-vegetarian and vegetarian respondents did not smoke. The result implies that majority of the respondents are not exposed to smoking that can cause lung cancer in the long run. The status of the respondents with regard to cigarette smoking revealed that out of 12 non-vegetarian respondents who smoke, 83\% of them already quit from the habit while $17 \%$ of them were still smoking. For the vegetarian respondents, they had already quit smoking. The result suggests that majority of the respondents who smoke had already quit long before becoming senior citizens. This is supported in the study of Hutton (1994), which stated that non-smokers look younger than smokers with the same ages. Not only that, quitting smoking is beneficial for every individual because smoking can cause the blood supply to lessen that will eventually cause the wrinkling of the skin. There were $31 \%$ of the non-vegetarian respondents who were exposed to secondhand smoke while only $10 \%$ for the vegetarian respondents. $69 \%$ of the non-vegetarian were not exposed while $93 \%$ of the vegetarians were not exposed as well. The result showed a good indication that the respondents would live longer and enjoy life much longer being free from secondhand smoke as this may cause lung cancer. 


\subsection{Health Status}

Majority of the non-vegetarian respondents were clustered from below average to good cardiovascular endurance (73\%) while vegetarian respondents were clustered from very poor to excellent cardiovascular endurance (100\%). The results implied that there were vegetarian respondents who have excellent cardiovascular endurance while the non-vegetarian respondents have better cardiovascular according to the clustered below average to good cardiovascular endurance. It can be deduced that both the non-vegetarian and vegetarian respondents have good cardiovascular endurance; this is mainly attributed to the reason that majority of the respondents had already quit smoking prior to becoming senior citizens and were free from secondhand smoke that helped them to have better cardiovascular endurance. Majority of the non-vegetarian respondents' flexibility were clustered from very poor to good (73\%) while that of the vegetarian respondents were also clustered from very poor to good (97\%). The result implies that the vegetarian respondents have better flexibility than the non-vegetarians. In the study of Quinn (2007), it was found out that protein, iron, and B12 are essential in effective muscle flexibility and strength training performance and these are not so much present in vegetarian diet. In order for a vegetarian person to improve his flexibility, he/she should supplement himself/herself with vitamin B12. Thus, in this study majority of the vegetarian respondents have higher intake of vitamin B12 which aid them to have a better and higher flexibility than the non-vegetarians.

There were $26 \%$ of the non-vegetarian respondents who got a very poor muscular endurance, $14 \%$ got excellent, $10 \%$ got above average, another $10 \%$ got poor muscular endurance, $9 \%$ got good, and $4 \%$ got below average muscular endurance. As for the vegetarian respondents, $30 \%$ got an excellent muscular endurance, another $30 \%$ got very poor, $13 \%$ got good, another $13 \%$ got poor, $7 \%$ got above average, and another $7 \%$ got average muscular endurance. These show that more non-vegetarian respondents who had performed the activity got lower muscular endurance while the vegetarian respondents had better muscular endurance. This could be supported with the study of Husain (2009), that having a healthy lifestyle free from smoking and alcohol coupled with regular exercise and having a vegetarian diet increases muscular strength and joint support. Thus, in this study, it was prevalent that vegetarian respondents have better muscular endurance than those of the non-vegetarians.

Majority of the non-vegetarian respondents' muscular strength were clustered from below average to above average ( $49 \%$ ) while vegetarian respondents muscular strength were clustered also from below average to above average (84\%). Based on the clustered results of the respondents' muscular strength, it was found out that the vegetarian respondents have better muscular strength than the non-vegetarian respondents. This could be supported with the study of Barr and Rideout (2004) stating that well-planned, appropriately supplemented vegetarian diets appear to effectively support muscular performance.

The medical condition of the respondents revealed that more than half of the non-vegetarian respondents (71\%) were suffering from diseases/illnesses while $29 \%$ of them were not. For the vegetarian respondents, $70 \%$ of them were not suffering from any disease and $30 \%$ of them were suffering from different illnesses/diseases. It can be concluded based on the results that more than half of the total respondents (59\%) were suffering from a different disease or illnesses. It is confirmed by the study of DeYoung, et al., (2005) which says that aging is inevitable. As one grows older the body organs seems to weaken and its functions altered that includes taste and smell, saliva secretion, swallowing difficulties, liver function and intestinal function. Everyone will grow old but how an individual responds to these changes reflects health status across the life span. Thus, the role of nutrition during the life span categories of adolescence through the middle years provides sufficient information to predict body processes related to aging.

For most of the non-vegetarian respondents, arthritis (44\%) was the first common illness. Ranked second was high blood pressure, and ranked third was diabetes. The top diseases experienced by the vegetarian respondents were high blood pressure (ranked $1^{\text {st }}$ ), diabetes (ranked $2^{\text {nd }}$ ), and shared the $6^{\text {th }}$ rank were anemia, arthritis, bronchitis, cancer and kidney stones. As the body's metabolism starts to slow down, diseases and illnesses will begin to take its toll. Nevertheless, a person can withhold the toll of aging through proper and healthy diet. One study by Melina, Davis, and Harrison (2005), stated that a vegetarian diet is healthier than a diet that emphasizes animal foods. Their study found out that vegetarian diet reduces one's rick of chronic, degenerative diseases such coronary artery disease, cancer, diabetes, obesity, gallbladder disease, and hypertension. Thus, there were more non-vegetarian respondents who were suffering from diseases than those who were into vegetarian diet.

Majority of the non-vegetarian respondents (76\%) and vegetarian respondents (88\%) were suffering from their disease for $1-19$ years already. The average year that the non-vegetarian respondents had been suffering from their diseases was 15 years and 10 years for the vegetarian respondents. The results imply that the toll of aging is experienced by the respondents as DeYoung et al., (2005), stated that everyone ages differently and it all depends how 
the body would react with time. It now depends on how the person lives his younger years and the kind of diet he is into. Of the nine vegetarian respondents who were suffering from a disease, 5 got the diseases before adapting a vegetarian diet while 4 got the disease after adapting the diet. The results imply that adapting a vegetarian diet cannot be a guarantee that a person cannot acquire any diseases anymore, however, there are other factors that can contribute that causes a person to acquire one, for instance, inactivity or psychological aspects. Furthermore, Gordon (2008), stated that a person choosing vegetarian diet and do not have professional advice could cause him/her to be deficit in certain vitamins and minerals. Thus, a vegetarian diet done incorrectly would be downright unhealthy. Less than majority of the respondent were vegan ( 47\%) and macrobiotic ( 20\%). With these kinds of diet and based on the study of Anderson and Prior (2007), when followed carefully and with a dietitians' advise, vegetarian diet helps in lowering levels of cholesterol, saturated fats, and body weight. Further, it also reduces the risk of heart diseases, keeps blood pressure low, and lessened the chances of developing Type-2 diabetes. Thus, based on the result majority of the vegetarian respondents have thinner and leaner body structure.

\section{Findings}

Using the t-test, the results show that the vegetarian respondents have better muscular endurance and muscular strength than the non-vegetarian respondents while their cardiovascular endurance and flexibility did not differ at all. The difference in their muscular endurance and muscular strength could be attributed to the vegetarian diets of the vegetarian respondents. This result is supported with the study of Husain (2009), that a vegetarian diet of at least five servings of fruits and vegetables everyday coupled with regular exercise can lead to a better muscular strength, muscular endurance and joint support that is free from arthritis.

Using the Spearman's rho Coefficient $(\rho)$, the results demonstrate that vegetarian diets have a positive correlation with their cardiovascular endurance and muscle strength. Nevertheless, the type of respondents did not have any correlation with flexibility and posted a very low correlation with muscular endurance. This result can be supported with the study of Scharffenberg (2005). His study found out that vegetarian diet is low in cholesterol and saturated fat that can cause greater heart attack, thus, reducing the risk thereof. It was found out further that with lower cholesterol levels, it can increase cardiovascular endurance, muscular strength and muscular endurance.

Furthermore, it was found out that significant relationship exist between type of respondents and stress resiliency level and so with personality type. This signify that diet of the vegetarian respondent was positively correlated with personality type, stress resiliency level, and slightly correlated with socio-economic status. Moreover, this implies that vegetarian respondents have better personality type and stress resiliency level. The majority of the vegetarian respondents have higher socio-economic status. This invite the idea that health education is essential in becoming healthy nowadays. In order to fully maximize the effects of vegetarian diets, it is important to consult a dietitian and an individual must have enough financial provision to do so. The study of Didsdall (2002), found out that low-income groups of people have a greater tendency to practice unhealthy diets compared to higher socio-economic groups. Pierson (2008), on the other hand, asserted that without proper knowledge on vegetarian diet on the over-consumption of highcalorie vegetarian foods will lead to weight gain just like meat-based foods, thus, an individual should be educated first on the nutritional values before following a vegetarian regimen. Another factor affected with the type of respondents was resiliency and type of personality. Alejandro (2006), found out that the consumption of meat and other animal products leads to the increase of body temperature that has been conclusively linked to heart attack, thus, affecting the resiliency and personality of a person.

In addition, the results demonstrated that there exists a significant relationship with muscular endurance with smoking and drinking alcoholic beverages. Also, there exists a significant relationship between muscular strength and drinking. This means that the muscular endurance was negatively correlated with smoking and drinking; and muscular strength is negatively correlated with smoking. This implies that as the respondents will lessen or stop their cigarette smoking and drinking of alcoholic beverages, their muscle endurance and strength improve. The correlation of muscular endurance and muscular strength can be supported with the study of Nieman and Stanton (2005), they found out that cigarette smoking and too much drinking of alcoholic drinks can lead to coronary diseases that weaken the body muscles, thus lowering the muscle endurance and muscle strength. On the other hand, Hutton (1994) added that smoking can cause the wrinkling of one's skin that leads to poor flexibility and also leads to poor muscle endurance and strength. With the negatives effects of smoking and drinking, it can be concluded that to attain improved and better physical fitness, one must be free from vices.

The significant relationship between medical condition with personality type, stress resiliency level and active 
physical participation also exist. This means that the medical condition was positively correlated with stress resiliency level, personality type, and physical activity participation. The result further implies that the better the medical condition of the respondents, the better is its stress resiliency level, better personality type, and improved physical activity participation. Even though aging is inevitable, a person can withhold time by living a healthy lifestyle through vegetarian diet that leads to better medical condition. There are several studies conducted about vegetarian diets that can lead to healthier body. One study by Melina, Davis, and Harrison (2005), suggested that vegetarian diet reduces one's risk of chronic, degenerative diseases such as coronary artery disease, cancer, diabetes, obesity, osteoporosis, gallbladder disease and hypertension. With all the benefits of vegetarian diets, it can be concluded that vegetarian diets can lead to a better medical condition and improved physical activity participation.

Similarly, there exists a significant relationship between lifestyle diseases and stress resiliency level and so with physical activity participation. This result suggests that lifestyle diseases were negatively correlated with stress resiliency level, and personality type. This means that the better the personality type and stress resiliency level of the respondents, the lesser he/she will acquire the lifestyle diseases. DeYoung et al., (2005) in their study stated that the elderly may struggle with the deaths of family members and prone to emotional breakdown. He further added that sometimes death of family members to lead to isolation and depression. Thus, a person with a well-adjusted personality and high stress resiliency level has a lower risk of lifestyle diseases and may live longer.

\section{Conclusion}

The findings of this study lead to the conclusion that having a vegetarian diet is good; however, it is not a guarantee that a person will no longer acquire diseases. This is true since man's body is composed of cells which are tremendously fragile that needs extreme care. While body cells are healthy a person could function well. They are not seen in our naked eye; however, in times that they become weak the body can no longer perform well. The concept of taking some precautionary measures in order to avoid sickness is very important in one's life. Ultimately, its significance should be considered.

\section{References}

Alejandro, J. (2006). Go Vegetarian. Retrieved February 20, 2010 from http://www. piercingpens.net/isabel-roces-go-vegetarian

Barr, S.I. \& Rideout, C.A .(2004 ).Nutritional Considerations for Vegetarian Athletes. Retrieved February 1, 2010 from http://www.extremevegansports. org/ pages/evidence.htm

Cox, G. ( 2002 ).Special needs: the vegetarian athlete. retrieved November 9, 2009 from http://home.pacbell.net/epski/vegan/ studiesnutrition/VegAthlete.pdf

DeYoung, G. L., et al. ( 2005 ). Foundations and clinical application of nutrition: A nursing approach. Philippines: ELSEVIER (Singapore) PTE LTD, Third Edition

Didsdall, et al. (2000). Low-income consumers' attitudes and behavior towards access, availability and motivation to eat fruit and vegetables.

Dugang, A. (2001). The Green Years. Retrieved February 20, 2010 from http://www. filipinovegetarianrecipe.com/green_years.htm

Dworkin, N. (1999). 22 Reasons to go vegetarian right now - benefits of vegetarian diet. December 9, 2009 from http://findarticles.com /p/articles/mi_m0820/is_1999_April/ai_54232138/

Ewan, C. (2002). Effect of vegetarian diets on performance in strength sports. Retrieved December 9, 2009 from http://www.sportsci.org/jour/0201/cf-e.htm

Faloon, W. ( 2006). Do vegetarians live longer. LE Magazine. Retrieved December 3, 2009 from http://www.lef.org/magazine/ mag2006/jan2006_awsi_01.htm.

Gordon, S. (2008). Vegetarian diets: Myths vs facts. Retrieved February 20, 2010 from zzhttp://kingsoopers.staywellsolutionsonline.com/library/wellness/1,1876

Husain, S. (2009). Eat to beat illness/Arthritis. Retrieved January 5, 2010 from http://www. india diets.com/diets

Hutton, D. ( 1994). Vogue beauty for life. New York. Crown Publishers, Inc.

Melina, V, Davis,B. \& Harrison, V. What is Vegetarian?. The vegetarian advantage. Philippines Publishing House, pp. $24-26$.

Nieman, D. C. \& Stanton, H. J.(2005). The Adventist advantage: An alternative lifestyle. The vegetarian advantage. Philippines Publishing House, pp. $31-34$.

Ornish, M.D.(1993). Why is a 'vegetarian diet' more healthy and what diet plans have made this claim. Retrieved December 8, 2009 from http://www.amhrt. Heart and Stroke A A Z Guide/ornish.html

Phillips, F. (2005). Vegetarian nutrition. Retrieved December 10, 2009 from http://www. prijatelji-zivotinja.hr/data/file_4586.pdf

Pierson, D. (2008).Vegetarianism has many benefits. Retrieved November 9, 2009 from http://www.GoodHealthlnfo.info.

Quinn, E. (2007). Nutrition tips for vegetarian athletes. Retrieved January 7, 2010 from http://sportsmedicine.about.com/ 
od/sportsnutrition/a/VegetarianTips.htm

Rakesh, S. (2000). vegetarian v/s non-vegetarian. Retrieved December 8, 2009 from http://www.ourkarnataka.com/madhwakalyanal vegetarian.htm

Segasothy, M \& Phillips, P.A. (1999). Vegetarian diet: Panacea for modern lifestyle diseases? Retrieved December 8, 2009 from http://qjmed.oxfordjournals.org/cgi/content/full/92/9/531

Senior Citizens Act (Republic Act No. 7432). ( n.d. ) Retrieved November 19, 2009 from http://www.dswd.gov.ph/

Siebert, Al. ( 2006). Type A Personality Test. Retrieved December 8, 2009 from http://discoveryhealth.queendom.com Iquestions/type_a_personality_1.html

Scharffenberg, J. (2005. Change your diet now. The vegetarian advantage. Philippines Publishing House, pp. 18 - 20.

Vance, F. Archbold, E. E. \& Cherne, H.M. (2004). Natural Remedies Encyclopedia. Fourth Edition. Harvestime Books. 